\title{
Development of a Microfluidic
} GHz Impedance Cytometer

\author{
Entwicklung eines mikrofluidischen GHz Impedanz-Zytometers
}

Niels Haandbæk*, Sebastian C. Bürgel, Dept. of Biosystems Science and Engineering, ETH Zurich, Basel, Flavio Heer, Zurich Instruments AG, Zurich, Andreas Hierlemann, Dept. of Biosystems Science and Engineering, ETH Zurich, Basel

* Correspondence author: niels.haandbaek@bsse.ethz.ch

Summary This article presents a novel microfluidic impedance cytometer enabling dielectric characterization of single cells at frequencies up to $500 \mathrm{MHz}$. The dielectric properties of cells at lower frequencies contain information about their size and membrane capacitance. The increased frequency range of the presented cytometer potentially allows for characterization of intracellular components, such as vacuoles or the cell nuclei. We demonstrate the overall capabilities of the cytometer through discrimination of polystyrene beads from Chinese hamster ovary $(\mathrm{CHO})$ cells. The discrimination is based on the difference in dielectric properties at frequencies up to $500 \mathrm{MHz}$. Artikel wird ein neuartiges mikrofluidischer Impedanz Zytome- ter vorgestellt, das die Charakterisierung der dielektrischen Eigenschaften von einzelnen Zellen bei Frequenzen von bis zu $500 \mathrm{MHz}$ ermöglicht. Die dielektrischen Eigenschaften von Zellen beinhalten bei niedrigen Frequenzen Informationen sowohl über die Zell-Größe als auch über die MembranKapazität. Der erhöhte Frequenzbereich des Zytometers ermöglicht potenziell die Charakterisierung von intrazellularen Bestandteilen, wie zum Beispiel Vakuolen und Zellkernen. Wir demonstrieren die Leistungsfähigkeiten des Zytometers, indem wir Polystyren-Teilchen von Zellen aus Ovarien des chinesischen Hamsters (CHO) differenzieren. Die Differenzierung basiert auf verschiedenen dielektrischen Eigenschaften der Partikel und Zellen bei Frequenzen bis $500 \mathrm{MHz}$.

Keywords Microfluidic impedance cytometer, single-cell analysis, impedance spectroscopy, high-frequency lock-in amplifier, multi-frequency analysis, CHO cells $\gg$ Schlagwörter Mikrofluidischer Impedanz Zytometer, Einzelzellenanalyse, Impedanzspektroskopie, Hochfrequenz Lock-in-Verstärker, Multifrequenzanalyse, CHO Zellen

\section{Introduction}

Impedance spectroscopy is a non-invasive technique for measuring the dielectric properties of a material of interest. The technique is often used in the area of material science, as reviewed in [1], but has recently also gained relevance for the characterization of biological tissues and cells, as reviewed in [2]. The dielectric properties of cells include information on membrane resistance, membrane capacitance and cytoplasmic conductivity at frequencies below $10 \mathrm{MHz}$ [3]. Intracellular features, such as the presence or size of vacuoles, can also potentially be detected, but at much higher frequencies [4].
Typically, bulk suspensions have been used for characterizing cells [4], and the results represent a population average [5]. The population average does not show important details about the heterogeneity of individual cells, such details, however, are needed to develop mathematical descriptions of cellular behavior. Analysis techniques featuring single-cell resolution have, therefore, become increasingly important [6].

The combination of impedance spectroscopy with a microfluidic flow cytometer results in a lab-on-chip device, capable of label-free analysis of single cells [7]. Cells or particles are dispersed in a liquid, typically an 
electrolyte, such as phosphate-buffered saline (PBS), and forced through a microfluidic channel with two pairs of planar electrodes patterned at the top and bottom. An AC voltage is applied to the electrodes causing a current flow. The current change upon passage of a particle is measured differentially and analyzed to determine the dielectric properties of the material between the electrodes.

Gawad et al. showed how different cell properties influence the impedance spectrum [8]. They found that the cell size contributes to the response at all frequencies, the membrane capacitance at frequencies around $1 \mathrm{MHz}$, and the cytoplasm conductivity at frequencies approaching $10 \mathrm{MHz}$. Cheung et al. used a similar setup with simultaneous analysis of multiple frequencies to discriminate beads from red blood cells and red-blood-cell ghosts [9]. Schade-Kampmann et al. used an impedance device for not only discriminating different cell types, but also for analyzing viability and apoptosis of Jurkat cells [10]. Holmes et al. used the method for discriminating different leukocytes in human blood [11]. They were able to make a differential white blood cell count with results that correlated well to those of commercial blood analyzers. Impedance work of other authors has been reviewed by Sun et al. [12].

In this paper, we describe a microfluidic impedance cytometer that covers a frequency range from DC to up to $500 \mathrm{MHz}$. The increased frequency range will allow for characterization of intracellular components, such as vacuoles and cell nuclei, in addition to the properties that are detectable at lower frequencies. The cytometer consists of an impedance spectroscope, combined with a simple microfluidic device with facing electrodes. The impedance spectroscope is based on a flexible FPGA platform, which interfaces to the microfluidic device and features highspeed data converters and a trans-impedance amplifier for current-to-voltage conversion that has been built from off-the-shelf components. We evaluate the electrical performance of the impedance spectroscope. We also demonstrate the capabilities of the cytometer in discriminating polystyrene beads from Chinese hamster ovary $(\mathrm{CHO})$ cells, based on the difference in their dielectric properties at frequencies up to $500 \mathrm{MHz}$.

\section{Materials and Methods}

\subsection{Microfluidic Impedance Cytometer}

A schematic diagram of the cytometer is shown in Fig. 1a. It consists of an impedance spectroscope combined with a microfluidic device. The impedance spectroscope is based on a UHFLI lock-in amplifier (Zurich Instruments AG, Switzerland), which generates the stimulation voltage and analyses the response current. A power amplifier (PA) is used to buffer the signal of the impedance spectroscope before it is applied to the microfluidic device. This is necessary because the impedance of the microfluidic channel is highly frequency dependent. A C2V converter $(\mathrm{C} 2 \mathrm{~V})$ transforms the response current into a voltage, which can be measured by the impedance spectroscope. $\mathrm{PA}$ and $\mathrm{C} 2 \mathrm{~V}$ are both implemented on a dedicated printed-circuit board (PCB) that also hosts the microfluidic device.

Figure $1 \mathrm{~b}$ illustrates the shape of the real, $\mathrm{X}$, and imaginary, Y, part of the differential voltage measured by the impedance spectroscope as a particle passes between the electrodes. $\mathrm{X}$ and $\mathrm{Y}$ are also known as the in-phase and out-of-phase components of the measured signal. The differential measurement gives rise to the distinct shape of $\mathrm{X}$ and $\mathrm{Y}$, which both consist of two opposite-sign Gaussian-like peaks. The extrema correspond to the time when the particle passes the center of each electrode. The amplitude of $\mathrm{X}$ and $\mathrm{Y}$ at the extrema is related to the

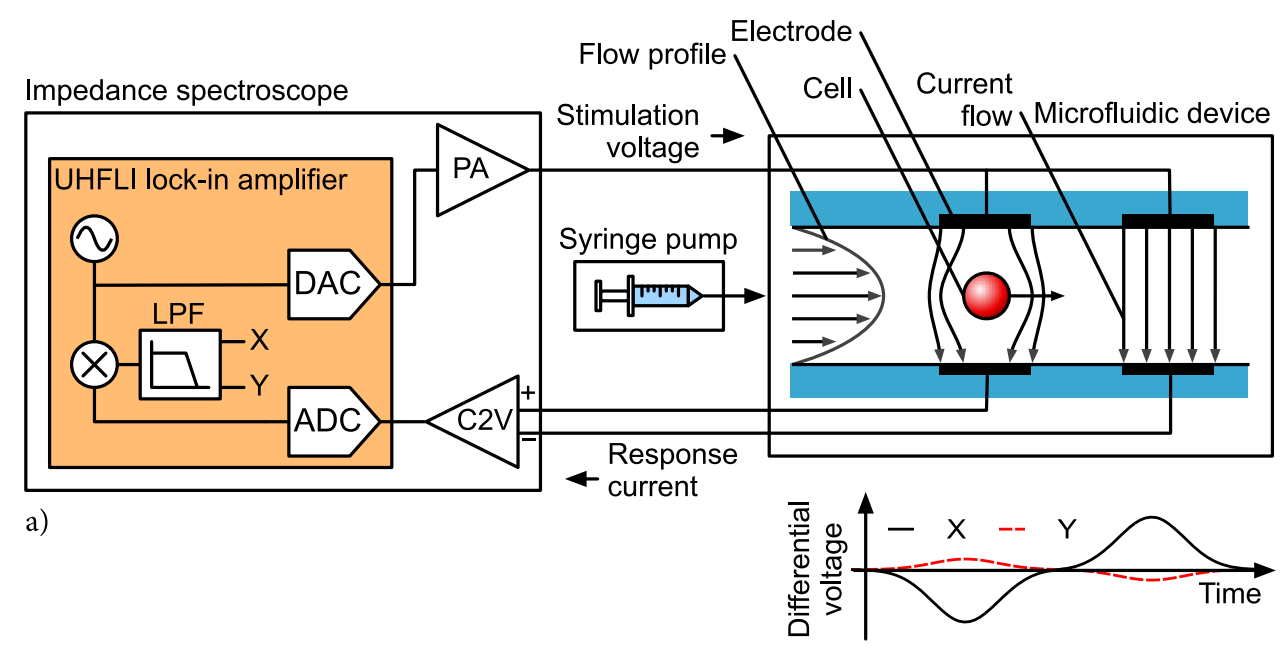

b)

Figure 1 (a) Schematic diagram showing the microfluidic impedance cytometer presented in this paper. A power amplifier (PA) buffers the signal from the impedance spectroscope, and a current-to-voltage converter (C2V) transforms the response current into a voltage. (b) Example of an analyzed signal as a cell or particle passes the electrode. 
dielectric properties of the particle. The time interval between the extrema can be used to determine the particle velocity.

\subsection{Lock-In Amplifier}

The UHFLI lock-in amplifier is shown in Fig. 2a. The connectors on the front panel are, from left to right, two high-frequency single-ended analog inputs, two highfrequency analog outputs, two reference inputs and four auxiliary outputs. The lock-in amplifier analyzes the signals on the high-frequency inputs, and the internal oscillators are driven to the high frequency outputs. In this work, only one input and one output has been used. The reference inputs may be used as external sources of reference oscillator signals. The auxiliary outputs display the in-phase and out-of-phase results of the lock-in amplifier in analog form.

Figure $2 \mathrm{~b}$ shows a block diagram of the UHFLI lock-in amplifier. Control and signal processing is implemented on a Xilinx Virtex-6 FPGA (Xilinx, Inc., USA). The input signals pass through a pair of variable-gain amplifiers (VGA), followed by anti-aliasing filters (AAF), before being sampled by the analog-to-digital converters (ADC) at 1.8 GSamples/s. The VGAs amplify the input signals with a maximum gain of $40 \mathrm{~dB}$ in order to allow the dynamic range of the ADCs to be used optimally. The AAFs are low-pass filters with a cut-off frequency of $600 \mathrm{MHz}$, which are needed to ensure that the frequency content of the input signals is limited to the Nyquist frequency of the ADCs. The output signals are first converted to the analog domain by means of two digital-to-analog converters (DACs), which also operate at 1.8 GSamples/s. Afterwards, they pass through two amplifiers, which are used to implement two different voltage amplitude ranges at the outputs: $150 \mathrm{mV}$ and $1.5 \mathrm{~V}$.

The lock-in amplifier enables generation and analysis of up to eight frequencies in parallel by means of eight reference oscillators and demodulators. Each demodulator operates on one of the two high-frequency inputs, which can be selected by means of a multiplexer (IMUX). The reference oscillator signal can be chosen freely from one of the eight digitally controlled oscillators (DCO) by means of another multiplexer (OMUX). The input and reference oscillator signals are combined in a complex mixer (MIX), and then low-pass filtered using two filters $(\mathrm{LPF})$. The samples of the resulting in-phase $(\mathrm{X})$ and
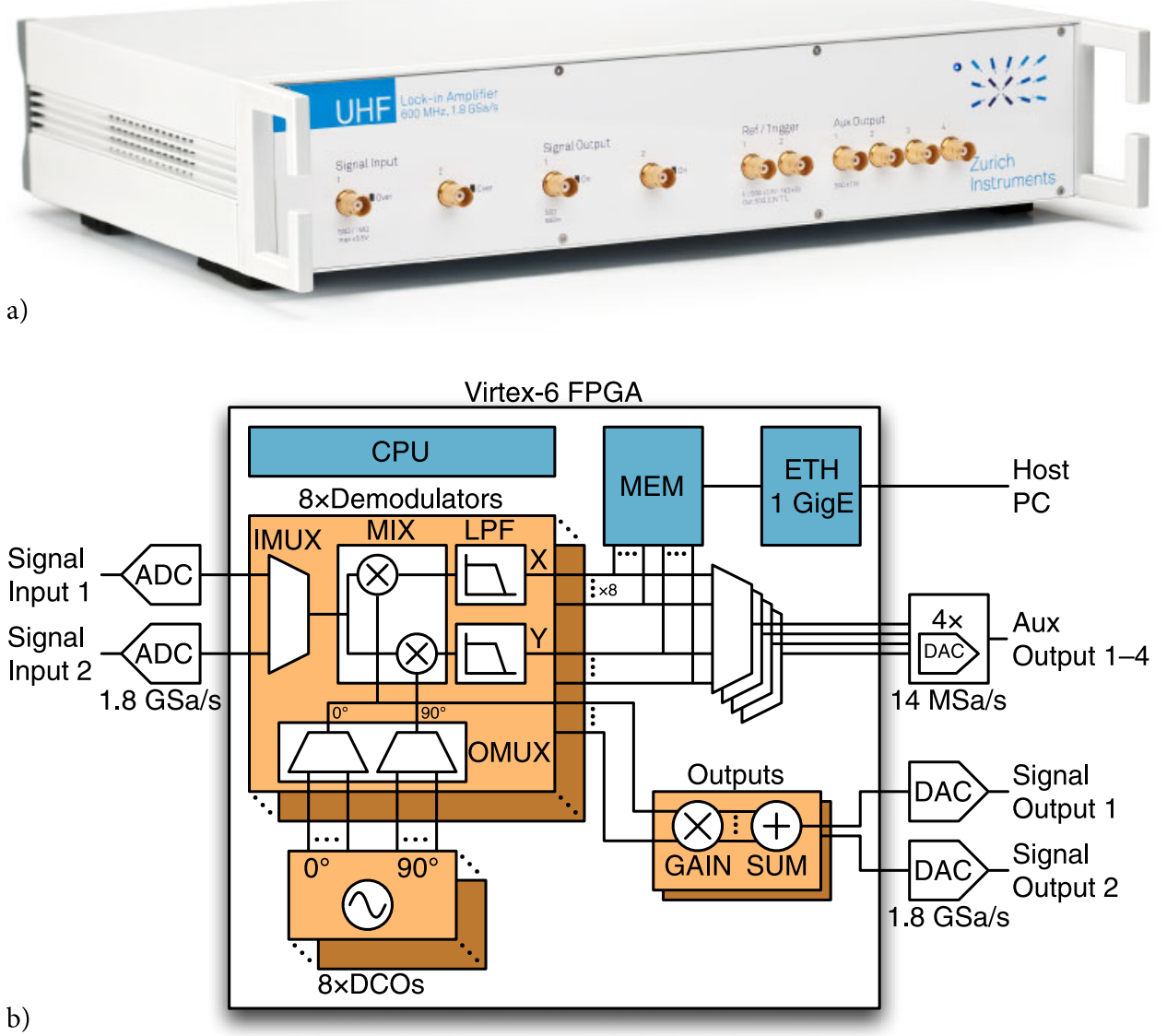

Figure 2 (a) Chassis of the UFHLI lock-in amplifier. The lock-in amplifier features two high-frequency inputs and outputs as well as the possibility to give out the analysis results in analog form through the auxiliary outputs. (b) Block diagram of the UHFLI lock-in amplifier with details of one of the eight demodulators. 
$90^{\circ}$-out-of-phase $(\mathrm{Y})$ signals are continuously buffered in the memory (MEM), packaged in User Datagram Protocol packets and streamed to the host PC over Ethernet (ETH). Here, they are stored for off-line analysis. The lock-in amplifier can reliably deliver samples at a combined sampling rate of all eight demodulators of up to 1.6 MSa/s. A microcontroller (CPU) controls the entire device and manages the overall communication with the host PC.

The reference oscillator signals of the demodulators are also used to drive the high-frequency outputs of the lockin amplifier. The reference signals are combined using a gain and summation operation. The gain of each signal can be independently controlled.

The signal processing principle is similar to that of conventional analog lock-in amplifiers, albeit implemented in the digital domain. The DCOs generate the phase of the digital sinusoidal reference oscillator signals by means of counters that increment at the sampling rate of the system within the range of $[0,2 \pi[$. The counters wrap around on overflow. The phase increment of each DCO is given by:

$\Delta \varphi=2 \pi \frac{f_{\mathrm{dco}}}{f_{s}}$,

where $f_{s}$ is the sampling rate of the system, i.e. $1.8 \mathrm{GHz}$, and $f_{\text {dco }}$ is the frequency of the reference signal, which falls in the range of $0 \mathrm{~Hz}$ to $600 \mathrm{MHz}$ with a resolution of better than $10 \mu \mathrm{Hz}$. The output of a DCO at time $n$ can be written as

$\Phi(n)=(n \Delta \varphi) \bmod 2 \pi$,

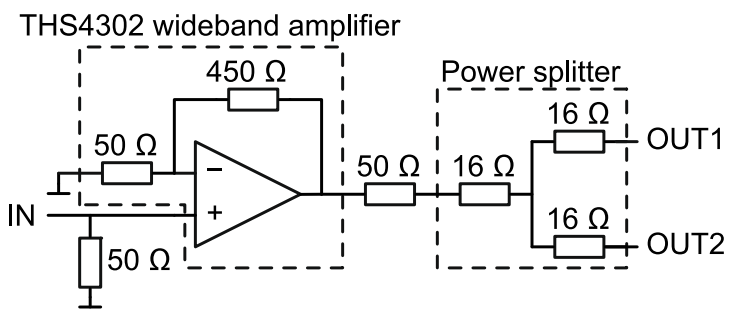

a)

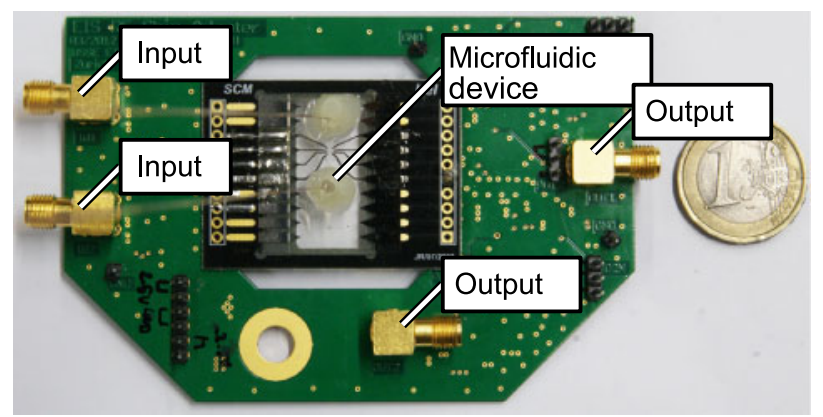

c) under the assumptions that $f_{\text {dco }}$ is constant and $\Phi(0)=0$. The actual sinusoidal reference oscillator signal is generated using two look-up tables (COS and SIN), which compute $\cos (\Phi(n))$ and $\sin (\Phi(n))$. The complex mixer in turn shifts the frequencies of the input signal, $f_{\text {in }}$, to $f_{\text {in }}-f_{\text {dco }}$ and $f_{\text {in }}+f_{\text {dco. }}$. The low-pass filters suppress the frequency components at $f_{\text {in }}+f_{\text {dco }}$, but also help to reject noise of the input signal. They feature configurable order, bandwidth and downsampling ratio to enable a flexible trade-off between noise rejection and response time.

\subsection{Power Amplifier and Current-to-Voltage Converter}

Figure 3a shows a schematic of the PA. It is based on a THS4302 wideband amplifier (Texas Instrument Inc., USA), which provides a gain of $14 \mathrm{~dB}$ up to a frequency of $1.8 \mathrm{GHz}$. The input impedance is matched to the $50 \Omega$ output impedance of the lock-in amplifier by means of a termination resistor, which results in a gain of $-6 \mathrm{~dB}$. A resistive power splitter at the output of the amplifier divides the output signal evenly between the two electrodes of the microfluidic device. The total gain, $G_{\mathrm{PA}}$, of the PA is $8 \mathrm{~dB}$ as long as it drives a high-impedance load such as the one of the microfluidic device.

Figure $3 \mathrm{~b}$ shows a schematic of the $\mathrm{C} 2 \mathrm{~V}$, which consists of two stages. The first stage is again based on a THS4303 amplifier. It is responsible for converting the response currents from each electrode into voltages. The second stage implements a differential-to-single ended conversion of the voltages by means of an ADA4927 differential amplifier (Analog Devices Inc., USA). The amplifier is

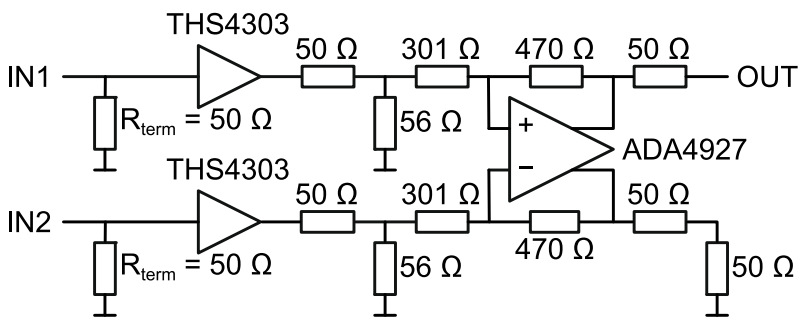

b)

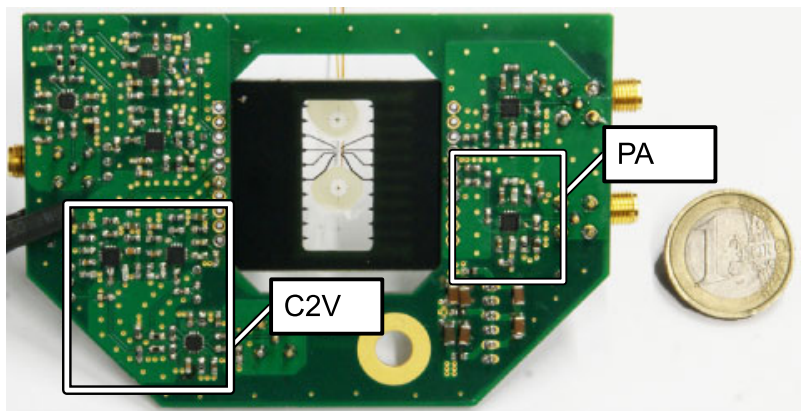

d)

Figure 3 (a) Schematic diagram of the PA. (b) Schematic diagram of the C2V converter. (c) Top of the PCB, which includes the PA, C2V and the microfluidic device. (d) Bottom view of the PCB with the components of a single PA and C2V being marked. 
configured with a gain of $3.7 \mathrm{~dB}$ by means of the feedback resistors of $470 \Omega$ and the gain resistors of $301 \Omega$. The output of the amplifier is matched to the input impedance of the lock-in amplifier by means of a $50 \Omega$ resistor. The unused output is grounded through an impedance of $100 \Omega$, which ensures that both outputs are loaded in a balanced fashion. The effective gain, $G_{\mathrm{C} 2 \mathrm{~V}}$, of the $\mathrm{C} 2 \mathrm{~V}$ is approximately $5.8 \mathrm{~dB}$, when it drives a $50 \Omega$ load such as that of the lock-in amplifier input.

Two pairs of PAs and C2Vs are integrated on a dedicated PCB, which is shown in Fig. $3 \mathrm{c}$ and $3 \mathrm{~d}$. The PCB includes a connector for the power supply of $\pm 2.5 \mathrm{~V}$. The PCB also holds the microfluidic device, which is mounted above a cutout that allows for observation of the microfluidic channel using an inverted microscope. The integration of the amplifiers and the microfluidic device on the same PCB allows the length of the connections between the components to be minimized, which reduces electrical reflections and, therefore, improves the signal quality.

\subsection{Microfluidic Device}

The microfluidic device, which is shown in Fig. 4, has been fabricated as described in [13]. It consists of two glass plates with 200 -nm-thick and 18 - $\mu$ m-wide platinum electrodes. The two plates are bonded face to face using a 40 - $\mu \mathrm{m}$-thick lithographically structured layer of SU-8 3000 as spacer in between that defines the channel dimensions. The final channel cross-section in the sensing region is $40 \times 40 \mu \mathrm{m}^{2}$. The microfluidic device features two sets of measurement electrodes with different dimensions.

\subsection{Cell and Bead Preparation}

For the experiment, CHO-K1 cells (CCL-61, American Type Culture Collection, USA) were cultured using standard conditions in Dulbecco's modified Eagle's medium with the addition of $10 \%$ fetal bovine serum (both from Life Technologies Europe B.V., Switzerland) and $1 \%$ penicillin-streptomycin. The cells were trypsinized and re-suspended in PBS (Sigma-Aldritch, Switzerland) to achieve a final concentration of approximately
100000 cells $/ \mathrm{ml}$. A suspension of $10-\mu \mathrm{m}$ polystyrene beads (Sigma-Aldrich, Switzerland) in PBS featuring a concentration of approximately 600000 beads/ml was also prepared. The two solutions were mixed and driven through the channel in the microfluidic device using a syringe pump (cetoni $\mathrm{GmbH}$, Germany) at a flow-rate of $5 \mu \mathrm{l} / \mathrm{min}$.

\subsection{Data Acquisition and Analysis}

The signal-to-noise ratio of the measurement at a given frequency is directly related to the amplitude of the output signal of the lock-in amplifier. In order to ensure that each frequency could be analyzed with a sufficient signalto-noise ratio, the measurement was limited to using only four of the eight demodulators. This enabled each frequency to be generated with an amplitude of $62.5 \mathrm{mV}$, which corresponds to $44.2 \mathrm{mV}_{\text {rms }}$. The use of even higher amplitudes leads to clipping at the output of the power amplifier. The four analysis frequencies where chosen so as to enable measurements of the cell size $(0.5 \mathrm{MHz})$, the membrane properties $(5 \mathrm{MHz})$, and intracellular components of the cell $(50 \mathrm{MHz}$ and $500 \mathrm{MHz})$. The response current was analyzed by using 3rd order low-pass filters with a bandwidth of $10 \mathrm{kHz}$. The resulting time-domain impedance signals were recorded with a sampling rate of $54.7 \mathrm{kSamples} / \mathrm{s}$ on the host PC using custom software (Zurich Instruments AG, Switzerland). The sampling rate was chosen to be higher than the Nyquist rate, which is two times the filter bandwidth, in order to avoid aliasing.

The time-domain impedance signals, an example of which is shown in Fig. 1, were analyzed to detect the particles and cells using MATLAB (MathWorks, Inc., USA). The in-phase part of the $0.5 \mathrm{MHz}$ signal was used to identify the exact timing of the individual peaks, because it exhibited the highest signal-to-noise ratio. First, all possible peaks were detected by comparing the absolute value of the in-phase part of the signal after subtraction of the DC offset to a fixed threshold. Next, the detected peaks were sorted according to polarity, i. e. positive or negative peaks, and grouped into pairs consisting of a negative peak and the following positive peak, which has been

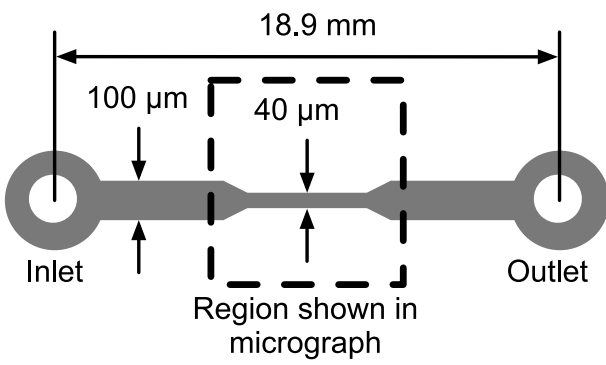

a)

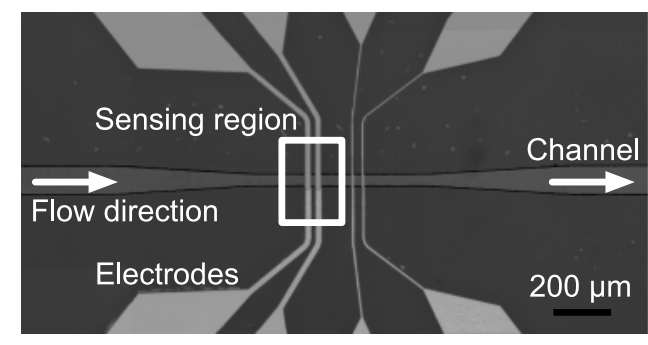

b)

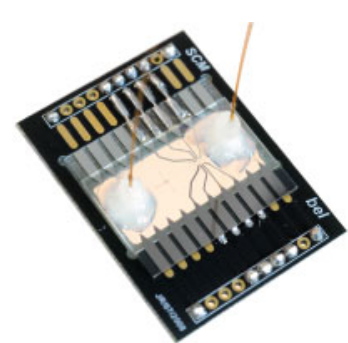

c)

Figure 4 (a) Schematic showing an overview of the microfluidic device. (b) Micrograph of the area around the sensing region of the microfluidic device. The electrodes are $18 \mu \mathrm{m}$ wide with a center-to-center spacing of $36 \mu \mathrm{m}$. (c) Microfluidic device with glass capillaries attached to inlet and outlet, mounted on a carrier PCB, which provides the electrical connections to the electrodes. 
assigned an "event". The timings of the peaks identified in this way were then used to extract the corresponding peak amplitudes at all other analyzed frequencies. Finally, peak-to-peak voltages were calculated by subtraction of the complex amplitude of the first and the second peak of each event for each analysis frequency.

In general, the impedance signals were dependent on size as well as position of the object within the channel. Size and channel position effects can, to some extent, be compensated by considering the opacity, which is given by

$$
O_{i}(f)=\frac{Z_{i}(f)}{Z_{i}(0.5 \mathrm{MHz})}
$$

where $Z_{i}(f)$ is the complex peak-to-peak voltage of particle $i$ measured at frequency $f$, and $O_{i}(f)$ is the resulting opacity.

The opacity values were further used for automatic discrimination between beads and cells by means of Fuzzy c-means clustering [14]. This algorithm only needs the number of clusters as input and otherwise operates in an unsupervised manner. Its advantage in comparison to the k-means algorithm is that it gives better results for overlapping events, which is advantageous for analyzing impedance data.

The input to the algorithm consists of the number of clusters, $c$, as well as the set of all detected events: $X=\left\{x_{1}, x_{2}, \ldots, x_{n}\right\}$, where $n$ is the number of detected events, and $x_{i}$ is a vector constructed from the real and imaginary parts of $O_{i}(f)$ for each frequency. The number of clusters was set to two in order to classify each event as either "cell" or "bead". The output of the algorithm consists of the set of cluster centers: $V=\left\{v_{1}, v_{2}, \ldots, v_{c}\right\}$ and a matrix $U$, of dimensionality $n$ by $c$, in which each element, $u_{i j} \in[0,1]$, represents the membership of the $i^{\text {th }}$ event to the $j^{\text {th }}$ cluster center. Each event was assigned to the cluster for which it had the highest membership. Finally, all membership values were compared against a threshold of 0.9 , and the events below threshold were marked as "unclassified".

\section{Results and Discussion}

\subsection{Frequency Response}

The performance of the impedance spectroscope was evaluated by measuring the frequency response of the individual components, namely that of the lock-in amplifier, the PA and C2V. Each component should have a bandwidth of at least $500 \mathrm{MHz}$ to ensure that highfrequency signals are not excessively dampened. Also, the delay through the individual components should preferably lead to as little phase distortion as possible.

The frequency response of the lock-in amplifier was measured by inserting a $50 \Omega$ coaxial cable directly between the input and the output of the instrument. The output was configured to generate a signal with an amplitude of $150 \mathrm{mV}$. The frequency was swept logarithmically between $50 \mathrm{kHz}$ and $500 \mathrm{MHz}$, and the magnitude and phase of the input signal was recorded. The results are shown in Fig. 5a. The magnitude displays low ripple over the entire frequency range of $\pm 0.2 \mathrm{~dB}$. The phase is also constant from close to $0^{\circ}$ up to a frequency of $300 \mathrm{MHz}$. At higher frequencies the delay in the analog part becomes comparable to the period of the input signal, which results in a linear decrease in phase.

The frequency response of the PA is shown in Fig. 5b. It was measured by connecting the PA in series between the input and output of the lock-in amplifier. As seen from the figure, the magnitude shows the expected DC gain of close to $14 \mathrm{~dB}$ and a bandwidth greater than $500 \mathrm{MHz}$. The roll-off in phase at higher frequencies is, again, caused by the delay in the amplifier.

The frequency response of the $\mathrm{C} 2 \mathrm{~V}$ was measured in a similar fashion. The frequency responses from the noninverting input (IN1) and inverting input (IN2) to the single output are shown in Fig. 5c. As can be seen in the figure, both paths show a very similar magnitude response with a DC gain close to the expected value of $5.8 \mathrm{~dB}$ and a bandwidth of only $420 \mathrm{MHz}$. The lower than expected bandwidth is due to the performance of the AD4927 amplifier, which is slightly degraded when driving a $100 \Omega$ load, however, this does not significantly
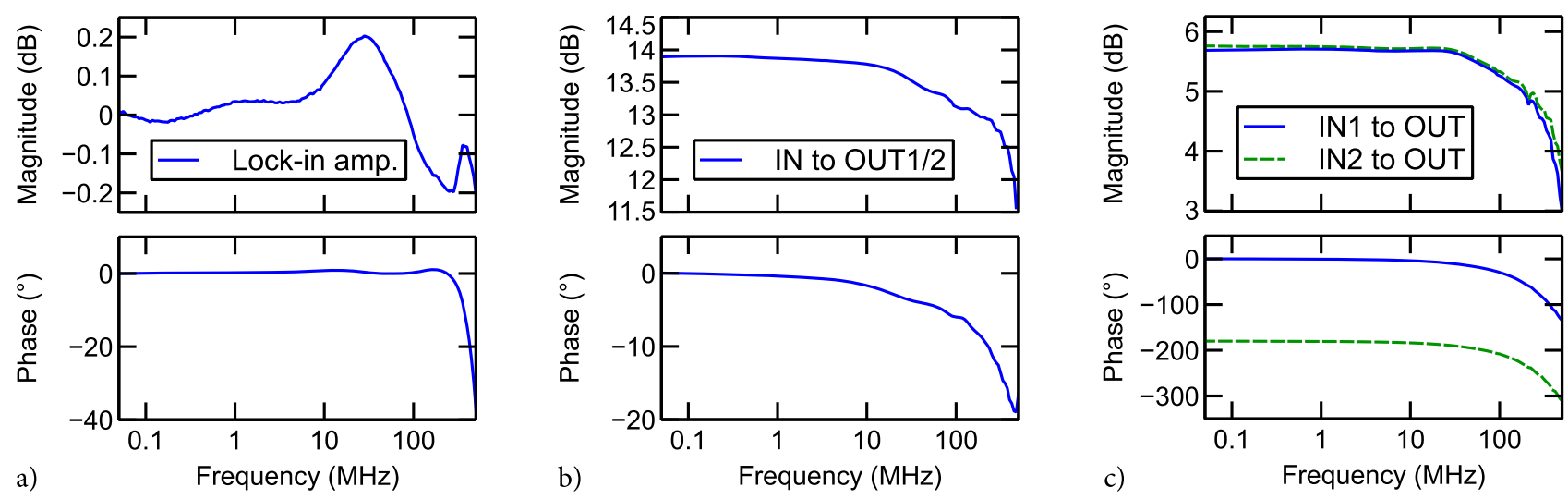

Figure 5 (a) Frequency response of the lock-in amplifier. (b) Frequency response of the PA from input to output. (c) Frequency response of the C2V for each path from input to output. The magnitude was normalized to the output voltage of $150 \mathrm{mV}$, and the phase was normalized to account for the extra delay introduced by the measurement cables. 
impact the performance of the system at high frequencies. The phase response shows the expected offset of $180^{\circ}$ for the inverting input.

\subsection{Noise and Sensitivity}

The sensitivity of the system is determined by the smallest signal change that it can detect, which is mainly determined by the noise of the electrical components. The most critical points in the system with respect to noise are those where the signal-to-noise ratio is lowest, namely the output of the $\mathrm{C} 2 \mathrm{~V}$ and the input of the lock-in amplifier. The power spectral density of the noise at these points is shown in Fig. 6.

As can be seen in the figure, the noise of lock-in am-

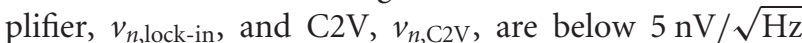
in the frequency range used for the experiments here. These values can be used to estimate the sensitivity of the system: First, the total noise at the input of the $\mathrm{C} 2 \mathrm{~V}$ is calculated for a measurement bandwidth, $f_{\mathrm{bw}}$, of $10 \mathrm{kHz}$, by root-squared addition of the two noise sources:

$v_{n, \text { total }}=\frac{\sqrt{f}_{\text {bw }}}{G_{\mathrm{C} 2 \mathrm{~V}}} \sqrt{v_{n, \text { lock-in }}^{2}+v_{n, \mathrm{C} 2 \mathrm{~V}}^{2}} \approx 180 \mathrm{nV}_{\mathrm{rms}}$

Conservatively, it is estimated that a minimum signal-tonoise ratio of $20 \mathrm{~dB}$ is needed for reliable characterization of cells or particles. The smallest detectable signal change at the input of the $\mathrm{C} 2 \mathrm{~V}$ is therefore:

$\Delta v_{\mathrm{C} 2 \mathrm{~V}, \min }=10 \cdot v_{n, \text { total }} \approx 1.8 \mu \mathrm{V}_{\mathrm{rms}}$

The actual signal at the input of the $\mathrm{C} 2 \mathrm{~V}$ can be approximated by:

$v_{\mathrm{C} 2 \mathrm{~V}}=v_{\text {out }} G_{\mathrm{PA}} \frac{R_{\mathrm{term}}}{Z_{\mathrm{ch}}}$

where $v_{\text {out }}$ is the output amplitude of the lock-in amplifier in Volts root-mean-square, $R_{\text {term }}$ is the termination resistor at the input of the $\mathrm{C} 2 \mathrm{~V}$, and $Z_{\mathrm{ch}}$ is the impedance

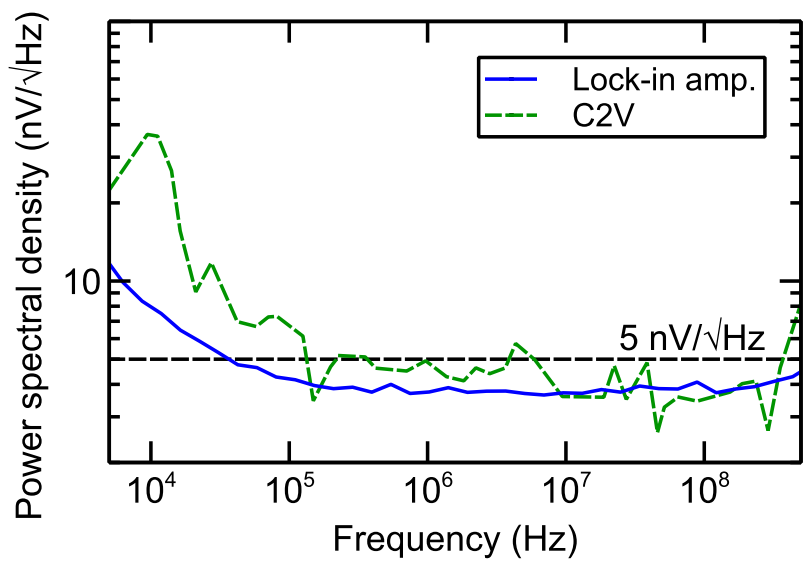

Figure 6 Power spectral density of the noise of the lock-in amplifier and the $\mathrm{C} 2 \mathrm{~V}$. The noise at lower frequencies is dominated by flicker noise, which is inversely proportional to the frequency. The flicker noise corner frequency is approximately $200 \mathrm{kHz}$. of the microfluidic channel at the chosen measurement frequency. Equations (6) and (7) can be used to find the smallest detectable impedance change in the channel, $\Delta Z_{\mathrm{ch}, \min }$

$v_{\mathrm{C} 2 \mathrm{~V}}-\Delta v_{\mathrm{C} 2 \mathrm{~V}, \text { min }}=v_{\mathrm{out}} G_{\mathrm{PA}} \frac{R_{\mathrm{term}}}{Z_{\mathrm{ch}}+\Delta Z_{\mathrm{ch}, \min }} \Leftrightarrow$

$\Delta Z_{\mathrm{ch}, \text { min }}=\frac{Z_{\mathrm{ch}} \Delta v_{\mathrm{C} 2 \mathrm{~V}, \text { min }}}{v_{\mathrm{out}} G_{\mathrm{PA}} \frac{R_{\mathrm{term}}}{Z_{\mathrm{ch}}}-\Delta v_{\mathrm{C} 2 \mathrm{~V}, \min }}$

In this example, it is assumed that the channel impedance is purely resistive and given by the physical dimensions of the channel combined with the conductivity of the medium, $\sigma_{\mathrm{PBS}}$, which is $1.6 \mathrm{~S} / \mathrm{m}$ :

$R_{\mathrm{ch}}=\frac{40 \mu \mathrm{m}}{\sigma_{\mathrm{PBS}} \cdot 40 \mu \mathrm{m} \cdot 18 \mu \mathrm{m}} \approx 35 \mathrm{k} \Omega$

This result, combined with the output voltage of $44.2 \mathrm{mVrms}$ and the termination resistor, $R_{\text {term }}$, of $50 \Omega$, results in a smallest detectable impedance change of:

$$
\begin{aligned}
\Delta R_{\mathrm{ch}, \min } & =\frac{R_{\mathrm{ch}} \Delta v_{\mathrm{C} 2 \mathrm{~V}, \min }}{v_{\mathrm{out}} G_{\mathrm{PA}} \frac{R_{\mathrm{term}}}{R_{\mathrm{ch}}}-\Delta v_{\mathrm{C} 2 \mathrm{~V}, \min }} \\
& =\frac{35 \mathrm{k} \Omega \cdot 1.8 \mu \mathrm{V}_{\mathrm{rms}}}{157 \mu \mathrm{V}_{\mathrm{rms}}} \approx 400 \Omega
\end{aligned}
$$

At low frequencies, the change in impedance is proportional to the volume of the detected object. If it is assumed that the object is spherical, a conservative estimate for the diameter of the smallest detectable object becomes:

$d_{\min }=2\left(\frac{3}{4 \pi} \frac{\Delta R_{\mathrm{ch}, \min }}{R_{\mathrm{ch}}} V_{\mathrm{ch}}\right)^{1 / 3} \approx 8.7 \mu \mathrm{m}$

where $V_{\mathrm{ch}}$ is the volume of the region between two measurement electrodes.

This analysis shows that the sensitivity of the impedance spectroscope, from an electrical point of view, is sufficient for the presented measurement, because beads and $\mathrm{CHO}$ cells are expected to have diameters larger than $10 \mu \mathrm{m}$. The sensitivity of the measurement can be improved either by increasing $R_{\text {term }}$ or by increasing the output voltage. It should be noted that an increase in $R_{\text {term }}$ also leads to an increase in noise, which is not captured by this basic formula. This limits the improvement in sensitivity that can be derived from an increase in $R_{\text {term }}$. It is also not possible to use arbitrarily large output voltages, as this may lead to an increase in electrochemical processes between electrodes and medium, which would render the measurements unstable.

\subsection{Impedance Results}

Figure $7 \mathrm{a}$ and $7 \mathrm{~b}$ show examples of the recorded impedance signals from the lock-in amplifier for $500 \mathrm{kHz}$ and $500 \mathrm{MHz}$, as a cell and a bead pass the electrodes. The figure shows that a significant impedance change can be detected for cells at frequencies of up to $500 \mathrm{MHz}$. The impedance signal exhibits a characteristic doublepeak, as the cell/bead moves through the sensing region, 

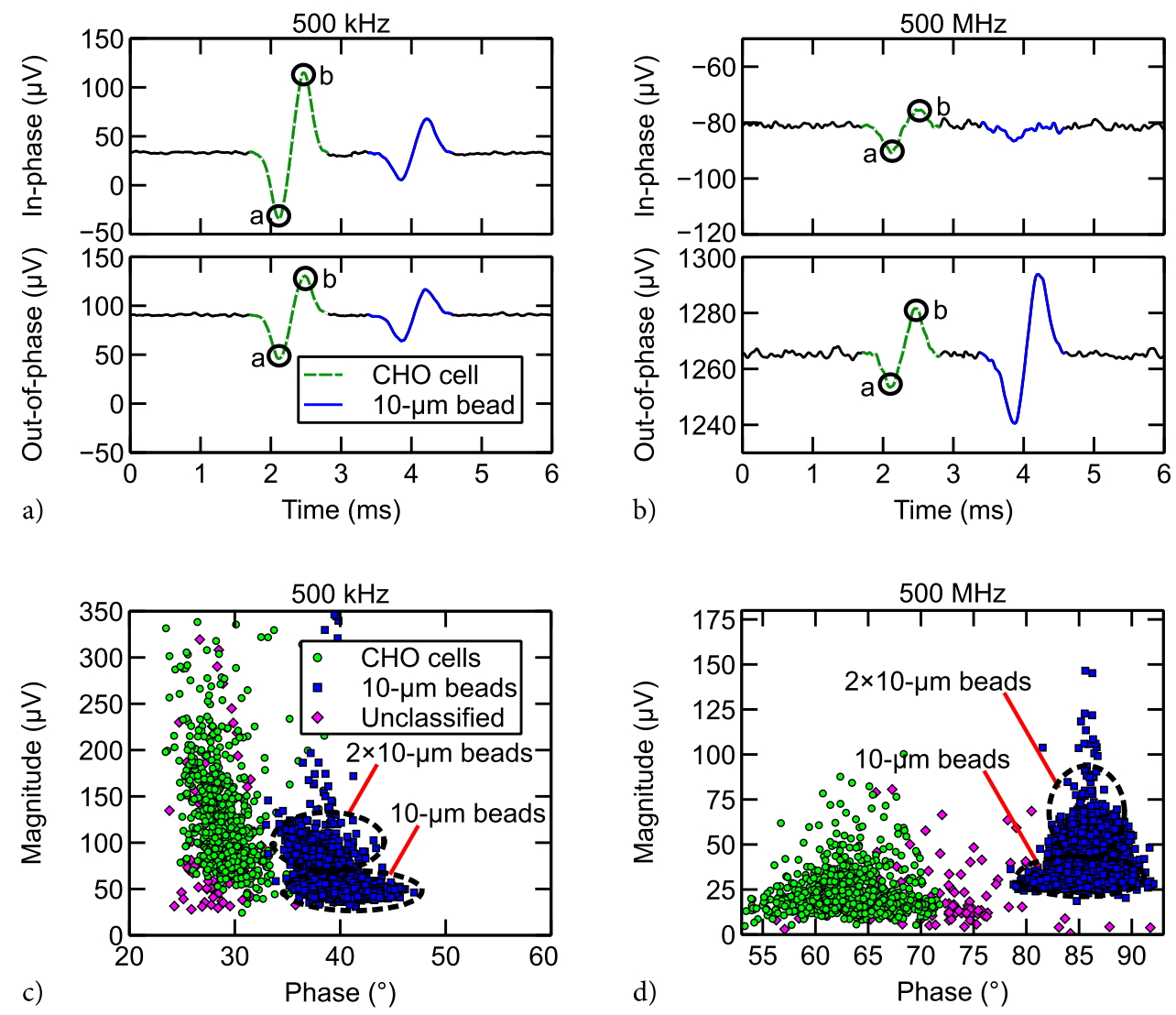

Figure 7 (a) Magnitude and phase of the recorded time-domain impedance signals at $500 \mathrm{kHz}$ showing peaks of a cell and a bead. (b) Corresponding time-domain signals at $500 \mathrm{MHz}$. (c) Impedance results of measurements at $500 \mathrm{kHz}$. (d) Impedance results at $500 \mathrm{MHz}$ showing distinct responses of cells and beads as a consequence of the difference in their dielectric properties.

which is a consequence of the impedance change and the differential measurement principle. Each double-peak was detected by the analysis algorithm, and a complex peak-to-peak value was calculated for each event by subtraction of the values at the points marked " $a$ " and " $b$ ". For example in Fig. $7 \mathrm{a} a=35 \mu \mathrm{V}-j 46 \mu \mathrm{V}$ and $b=-115 \mu \mathrm{V}-j 130 \mu \mathrm{V}$, which results in a peak-to-peak voltage of $a-b=150 \mu \mathrm{V}+j 84 \mu \mathrm{V}=172 \mu \mathrm{V} \angle 29^{\circ}$, where $j^{2}=-1$.

Figure $7 \mathrm{c}$ shows the magnitude and phase of the resulting complex peak-to-peak values for a complete measurement at $500 \mathrm{kHz}$. The coloring of the individual data points corresponds to the results of the automated discrimination analysis step. The magnitude of the impedance change at this frequency is mainly determined by the volume of the detected object. An overlap between cells and beads can be observed, which shows that they have comparable size. The average cell diameter is calculated to be $12.5 \mu \mathrm{m}$, which is close to reported values for CHO cells of $12.9 \mu \mathrm{m}$ [15]. A relatively large variation is to be expected, as no effort has been made to control the cell development stage during culturing. Two groups can be observed for the beads, which signify single beads and pairs of beads that stick together. The paired beads show the correspondingly larger impedance signal magnitude in comparison to single beads.
Figure $7 \mathrm{~d}$ shows a similar scatter plot for the results at $500 \mathrm{MHz}$. The impedance change at this frequency is dominated by the dielectric properties of cytoplasm and nucleus, which are comparable to those of the suspension medium. These cellular dielectric characteristics lead to lower amplitudes, and, therefore, smaller impedance changes, compared to those of non-conductive beads.

The phase signal of the impedance change is mainly determined by the capacitance of the detected object. The beads show a larger change at $500 \mathrm{kHz}$ and $500 \mathrm{MHz}$. This is due to the small relative permittivity of the polystyrene material, which causes a greater reduction in capacitance of the channel in comparison to cells.

Figure 8 a shows a scatter plot of the magnitude and phase of the opacity at $500 \mathrm{MHz}$. As seen from the figure, beads and cells cluster in two highly separated groups. In contrast to the raw impedance signals, magnitude and phase values of the opacity display Gaussian-like distributions. The two groups of single and paired beads are not visible in the plot, which makes it evident that the opacity better captures the dielectric properties of the detected objects regardless of their size. The opacity values also allow for good separation of beads and cells in magnitude and, to a lesser extent, in the phase in comparison to the raw impedance signal. This clearly shows that the beads remain opaque to the signal at high frequency, whereas 

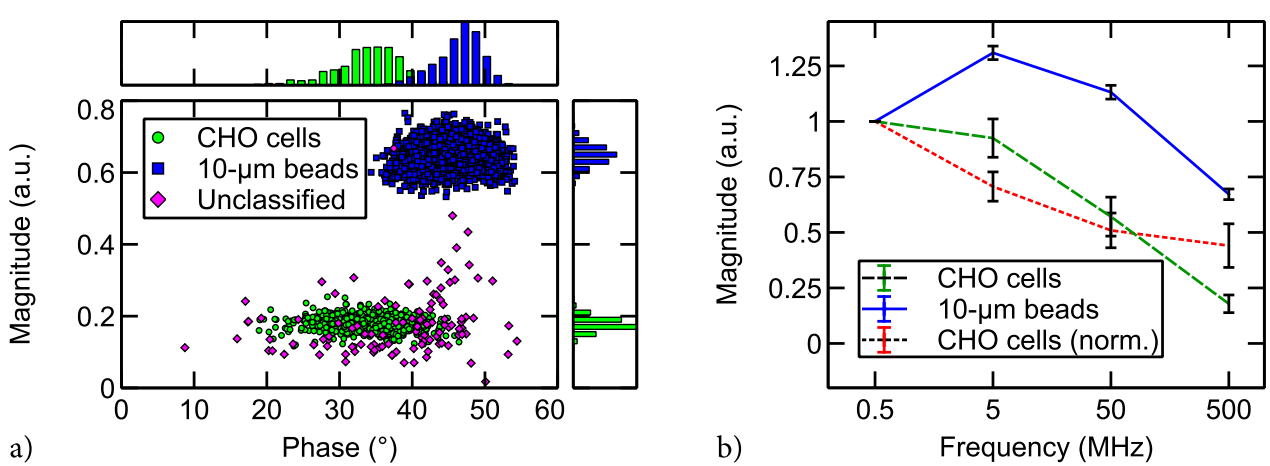

Figure 8 (a) Opacity magnitude and phase at $500 \mathrm{MHz}$. (b) Opacity magnitude at all four measured frequencies for beads and cells. The opacity at $0.5 \mathrm{MHz}$ is by definition one, because the impedance at this frequency is used as reference for the opacity value calculation. The "CHO cells (norm.)" curve is computed by normalizing the opacity of the cells to the mean opacity value of the beads.

the cells become translucent. The unclassified events are attributed to small particles, which have low signal-tonoise ratio, as well as to dead cells, which have dielectric properties between those of beads and cells.

Figure $8 \mathrm{~b}$ shows the magnitude of the opacity for all frequencies for beads and cells. The opacity has, by definition, the value 1 at $0.5 \mathrm{MHz}$, because this frequency is used as a reference for the opacity calculation. The opacity of beads is expected to be constant for all frequencies due to the uniform material, which should not show any frequency-dependent behavior within the range covered in this measurement. As can be seen in the figure, the opacity first increases at $5 \mathrm{MHz}$ and then decreases towards $500 \mathrm{MHz}$. The reason for the initial increase is that the signal at the reference frequency of $0.5 \mathrm{MHz}$ is slightly attenuated due to the existence of an electrical double layer (EDL) between the electrodes and the medium, which can be modeled as a capacitor. The slight decrease at higher frequencies can be attributed to the limited bandwidth of the impedance spectroscope.

The cells show a general decrease at all frequencies, which reveals little detail about their structure. However, the knowledge that the opacity of the beads should be constant at all frequencies can be used to compensate for the effects of the EDL as well as the limited bandwidth of the instrument. This is done by normalizing the opacity of the cells to the mean opacity of the beads, the result of which is represented by the "CHO cells (norm.)" curve. This curve displays a clear dispersion up to $50 \mathrm{MHz}$ due to the existence of the cell membrane. More analysis frequencies would be needed to reveal details about the internal structure of the cell.

\section{Summary and Outlook}

We have presented a novel microfluidic impedance cytometer, which includes an impedance spectroscope enabling simultaneous analysis at eight frequencies between DC and $600 \mathrm{MHz}$. The spectroscope features a more than ten-fold increase in the frequency range in comparison to other devices. The impedance spectroscope was constructed by using a high-frequency lock-in amplifier combined with an analog front-end, built from off-the-shelf components, consisting of a PA and a C2V converter. The electrical performance of the impedance spectroscope was characterized by measuring the frequency and noise responses of the lock-in amplifier and analog front-end. The frequency response showed very low ripple over the entire frequency range. The noise was measured to be lower than $5 \mathrm{nV} / \sqrt{\mathrm{Hz}}$ for both, the lock-in amplifier and $\mathrm{C} 2 \mathrm{~V}$, which is deemed sufficient for characterization of single-cells with diameters down to approximately $9 \mu \mathrm{m}$.

We demonstrated that the impedance cytometer is capable of measuring impedance characteristics of single $\mathrm{CHO}$ cells as well as of beads up to a frequency of $500 \mathrm{MHz}$. The results at $500 \mathrm{kHz}$ revealed the average cell diameter to be $12.5 \mu \mathrm{m}$, which is close to what has been reported by other authors. The results at $500 \mathrm{MHz}$ showed very distinct responses of cells and beads due to the difference in the respective dielectric properties. The cells gave rise to a smaller response in comparison to the beads. This was attributed to the comparably larger conductivity of the cell cytoplasm and nucleus, which dominates the response at high frequencies.

Current work is ongoing to use the high frequency capabilities of the device for characterizing cells according to differences in subcellular morphology. Examples include characterization of variations in mitochondrial morphology, which has been shown to play an important role in neurodegenerative diseases, as well as the detection of cells that have been infected by parasites.

\section{Acknowledgements}

This project received funding from the Commission for Technology and Innovation, CTI, Switzerland under project no. 11174.2 PFLS-LS.

\section{References}

[1] I. D. Raistrick, Application of Impedance Spectroscopy to Materials Science, Annual Review of Materials Science 16, 343-370 (1986); doi:10.1146/annurev.ms.16.080186.002015. 
[2] K. R. Foster and H.P. Schwan, Dielectric properties of tissues and biological materials: a critical review., Critical reviews in biomedical engineering 17, 25-104 (1989); PMID:2651001.

[3] R. Pethig and D. B. Kell, The passive electrical properties of biological systems: their significance in physiology, biophysics and biotechnology, Physics in Medicine and Biology 32, 933-970, (1987); doi:10.1088/0031-9155/32/8/001.

[4] K. Asami and T. Yonezawa, Dielectric behavior of wild-type yeast and vacuole-deficient mutant over a frequency range of $10 \mathrm{kHz}$ to $10 \mathrm{GHz}$., Biophysical journal 71, 2192-200 (1996); doi:10.1016/S0006-3495(96)79420-1.

[5] D. Di Carlo and L. P. Lee, Dynamic Single-Cell Analysis for Quantitative Biology, Analytical Chemistry 78, 7918-7925 (2006); doi:10.1021/ac069490p.

[6] H. A. Svahn and A. van den Berg, Single cells or large populations?, Lab on a chip 7, 544-546, (2007); doi:10.1039/b704632b.

[7] S. Gawad, L. Schild, and P. H. Renaud, Micromachined impedance spectroscopy flow cytometer for cell analysis and particle sizing., Lab on a chip 1, 76-82, (2001); doi:10.1039/b103933b.

[8] S. Gawad, K. Cheung, U. Seger, A. Bertsch, and P. Renaud, Dielectric spectroscopy in a micromachined flow cytometer: theoretical and practical considerations., Lab on a chip 4, 241-251 (2004); doi:10.1039/b313761a.

[9] K. Cheung, S. Gawad, and P. Renaud, Impedance spectroscopy flow cytometry: on-chip label-free cell differentiation, Cytometry. Part A?: the journal of the International Society for Analytical Cytology 65, 124-132 (2005); doi:10.1002/cyto.a.20141.

[10] G. Schade-Kampmann, A. Huwiler, M. Hebeisen, T. Hessler, and M. Di Berardino, On-chip non-invasive and label-free cell discrimination by impedance spectroscopy., Cell proliferation 41, 830-840 (2008); doi:10.1111/j.1365-2184.2008.00548.x.

[11] D. Holmes, D. Pettigrew, C. H. Reccius, J. D. Gwyer, C. van Berkel, J. Holloway, D. E. Davies, and H. Morgan, Leukocyte analysis and differentiation using high speed microfluidic single cell impedance cytometry., Lab on a chip 9, 2881-2889 (2009); doi:10.1039/b910053a.

[12] T. Sun and H. Morgan, Single-cell microfluidic impedance cytometry: a review, Microfluidics and Nanofluidics 8, 423-443 (2010); doi:10.1007/s10404-010-0580-9.

[13] S. C. Bürgel, Z. Zhu, N. Haandbaek, O. Frey, and A. Hierlemann, Dynamic and static impedance spectroscopy for single particle characterization in microfluidic chips, in 2012 IEEE 25th International Conference on Micro Electro Mechanical Systems (MEMS), 1033-1036 (2012); doi:10.1109/MEMSYS.2012.6170247.
[14] J.C. Bezdek, Pattern Recognition with Fuzzy Objective Function Algorithms. Boston, MA: Springer US (1981); doi:10.1007/978-14757-0450-1.

[15] W. Pilbrough, T. P. Munro, and P. Gray, Intraclonal protein expression heterogeneity in recombinant $\mathrm{CHO}$ cells., PloS one 4, e8432 (2009); doi:10.1371/journal.pone.0008.

Received: September 19, 2013, accepted: October 5, 2013

M. Sc. Niels Haandbæk studied electrical engineering at the Technical University of Denmark. He is currently pursuing a Ph.D. at the Department of Biosystems, Science and Engineering of ETH Zurich, in the field of impedance-based characterization of single cells.

Address: Department of Biosystems Science and Engineering (BSSE), Mattenstrasse 26, CH-4058 Basel, Switzerland,

e-mail: niels.haandbaek@bsse.ethz.ch

M.Sc. Sebastian C. Bürgel studied biomedical engineering and electrical engineering at ETH Zurich. He is currently working towards a Ph.D. at the Department of Biosystems, Science and Engineering of ETH Zurich, in the field of microdevices for impedance characterization of cells and tissues.

Address: See above

Dr. Flavio Heer studied physics at ETH Zurich where he received the Ph.D. degree in 2005. He is Co-founder and CTO of Zurich Instruments AG, Switzerland. As a specialist in analog circuit design and signal processing, he oversees the research and development of high-end instrumentation in Zurich Instrument's production line.

Address: Zurich Instruments AG, Technoparkstrasse 1, CH-8005 Zurich, Switzerland

Prof. Andreas Hierlemann received his Ph.D. in physical chemistry from the Eberhard-Karls University in Tübingen, Germany, in 1996. He was appointed full professor of "Biosystems Engineering" at the Department of Biosystems, Science and Engineering of ETH Zurich in Basel in 2008.

Address: Department of Biosystems Science and Engineering, Mattenstrasse 26, CH-4058 Basel, Switzerland 